\title{
Pedigree analysis of the EGFR p.V1010M germline mutation in a family with a family history of non-small-cell lung cancer
}

\author{
$\mathrm{Na} \mathrm{Li}^{1}$, Chunyi Liu ${ }^{1}$, Lan Xiong ${ }^{1}$, Depei Huang ${ }^{2} \wedge$, Youfan Jiang ${ }^{1}$ \\ ${ }^{1}$ Department of Respiratory Medicine, the Second Affiliated Hospital of Chongqing Medical University, Chongqing, China; ${ }^{2}$ The Medical \\ Department, 3D Medicines Inc., Shanghai, China \\ Contributions: (I) Conception and design: N Li, Y Jiang; (II) Administrative support: Y Jiang; (III) Provision of study materials or patients: C Liu, \\ L Xiong; (IV) Collection and assembly of data: C Liu, L Xiong; (V) Data analysis and interpretation: N Li, D Huang; (VI) Manuscript writing: All \\ authors; (VII) Final approval of manuscript: All authors. \\ Correspondence to: Youfan Jiang. Department of Respiratory Medicine, the Second Affiliated Hospital of Chongqing Medical University, Chongqing, \\ China. Email: jyf@hospital.cqmu.edu.cn.
}

Background: Tumors can be caused by genetic or environmental factors, but previous studies have shown that genetic factors contribute less to lung cancer than environmental factors. The epidermal growth factor receptor $(E G F R)$ is the most common driver gene in non-small-cell lung cancer (NSCLC), but most variations are somatic. In this study, we reported on the pedigree of the EGFR p.V1010M germline mutation for the first time, and explored the correlation between the V1010M and the occurrence of NSCLC. Further, the effect of the V1010M on the treatment of the EGFR-tyrosine kinase inhibitors (TKIs) was investigated through the treatment of the proband with the simultaneous somatic mutation of the EGFR p.L858R.

Methods: The family members were screened using next-generation sequencing (NGS) and Sanger sequencing, and the pedigree was analyzed to examine the relationship between the EGFR p.V1010M and the occurrence of NSCLC. Schrodinger software was used to predict the structural function of the mutant amino acid sequence proteins.

Results: A total of 10 blood samples were collected from 4 generations of family members, many of whom had suffered from lung cancer. Six carriers of the EGFR p.V1010M were detected. The pedigree analysis showed that there was still no evidence of a correlation between the EGFR p.V1010M and disease occurrence. Additionally, the proband had the EGFR p.L858R somatic mutation, and the response after the treatment of gifitinib was stable disease (SD), which turned to progressive disease (PD) some 4 months later. Schrodinger software showed that the 1010th amino acid valine was located near the $\mathrm{C}$ terminal, and the variation to methionine had little effect on the structure of the EGFR dimer.

Conclusions: This study is the first report on pedigree with the EGFR p.V1010M germline mutation. Further research needs to be conducted to determine whether this mutation is pathogenic, but it is likely related to EGFR-TKI resistance in NSCLC.

Keywords: Non-small-cell lung cancer (NSCLC); epidermal growth factor receptor p.V1010M (EGFR p.V1010M); germline; pedigree; resistance

Submitted Nov 10, 2021. Accepted for publication Jan 27, 2022.

doi: $10.21037 /$ atm-21-6925

View this article at: https://dx.doi.org/10.21037/atm-21-6925

^ ORCID: 0000-0003-1383-252X. 


\section{Introduction}

Malignant tumors are mainly caused by genetic, environmental, and other factors. The occurrence of malignant tumors is closely related to gene variation and epigenetics (1), which may be caused by a single driver gene variation or the cumulative effect of multiple gene variations. Additionally, both germline and somatic mutations are likely to cause cancer. Cancers can show high familial aggregation, but genetic factors may not play as dominant a role in tumorigenesis as previously thought. A study in the New England Fournal of Medicine in 2000 showed that the heritability of breast cancer, colorectal cancer, and prostate cancer was only $27-42 \%$ (2), meaning that environmental factors accounted for as much as $58-73 \%$ of the incidence of tumors.

Lung cancer has the highest morbidity and mortality rate in the world, and has an obvious familial aggregation. However, studies have shown that the heritability of lung cancer is even lower than breast cancer, colorectal cancer, and prostate cancer [about 26\% (2)], and the gene variation spectrum is completely different to that of other tumor species with high heritability. Lung cancer is most likely caused by single driver genes, such as epidermal growth factor receptor $(E G F R)$, anaplastic lymphoma kinase $(A L K)$, ROS proto-oncogene 1 (ROS1), and Kirsten rat sarcoma viral oncogene homologue (KRAS). Non-small-cell lung cancer (NSCLC) accounts for 80-85\% of lung cancers, and is the most successfully studied tumor species in current research on "precision therapy" for which treatment selection is based on molecular typing (1).

The EGFR is the most common driver gene of NSCLC and the major causes leading to NSCLC. Most of the EGFR mutations are somatic mutations, with a mutation rate of about $49.3 \%$ in the Asian NSCLC population (3), but only $0.11 \%$ are germline mutations (4). Its variation types are various, and are mostly located at exons $18-21$. The EGFR mutations could causes activation of the tyrosine kinase domain (TKD) by mimicking the conformational changes, which continuously activate downstream signaling pathways and ultimately lead to tumorigenesis (5). There have been 3 generations of EGFR-tyrosine kinase inhibitors (TKIs), of which exon 19 (19indel) and exon 21 (L858R) have the most accurate efficacy, and the variation of exon 18 and 20 generally have poor efficacy. Responses to EGFR-TKIs vary greatly from site to site, and each site needs to be analyzed separately (1).

Pedigree analysis is a method to analyze and judge the genetic pattern and transmission rule of a disease based on the incidence of a disease in the blood relationship diagram of several generations. This method is helpful to trace the disease in the family members of the first discovered patient-the proband as a clue, and make a comprehensive analysis to draw a conclusion about the genetic mode of the disease. In a Japanese study, pedigree analysis successfully showed a relationship between EGFR p.V843I germline mutations and lung cancer incidence (6).

In this study, we detected germinal EGFR mutation in a patient with lung cancer with obvious familial aggregation from clinical work, and made a larger pedigree after genetic screening of the family. The germline mutation EGFR p.V1010M was reported on for the first time. Its pathogenicity and drug sensitivity were examined, and the changes of protein spatial structure were predicted to provide data for a development mechanism and treatment plan for lung cancer.

We present the following article in accordance with the MDAR reporting checklist (available at https://atm. amegroups.com/article/view/10.21037/atm-21-6925/rc).

\section{Methods}

In our clinical work, we found a female lung cancer patient carrying EGFR germline mutation. The family members were screened using next-generation sequencing (NGS) and Sanger sequencing, and the pedigree was analyzed to examine the relationship between the EGFR p.V1010M and the occurrence of NSCLC. Schrodinger software was used to predict the structural function of the mutant amino acid sequence proteins. All procedures performed in this study involving human participants were in accordance with the Declaration of Helsinki (as revised in 2013). The study was approved by the Ethics Committee of the Second Affiliated Hospital of Chongqing Medical University (No. 135, 2021) and informed consent was taken from all the participants.

\section{Results}

\section{General information of the proband}

A female, aged 54 years old, who had never smoked, was admitted to a local hospital on June 5, 2019 complaining of having a "cough for 3 days". A mass in the posterior basal segment of the lower lobe of the right lung was detected, as were diffuse nodules and thickened interlobular septum in both lungs. There appeared to be a high possibility of right 
lower lobe peripheral lung cancer with bilateral pulmonary blood flow and lymph node metastasis, and obstructive pneumonia in right lower lobe. The patient visited our department on June 10, 2019, A bronchofiberscope biopsy revealed lung adenocarcinoma. Brain magnetic resonance imaging and a whole body bone scan showed no metastatic lesions, and the final diagnosis was right lung adenocarcinoma (T4N3M0, IIIc).

\section{Treatment process}

Gefitinib tablets were administered orally $[0.25 \mathrm{~g}$, every day (QD); see Figure 1]. No EGFR mutation was detected in the peripheral blood sample using the amplification refractory mutation system (ARMS), and an ADx-ARMS test kit (Amoy Diagnostics Co., Ltd., Xiamen, China) on the CFX96 Touch Real-Time PCR Detection System (BioRad, USA). However, on July 15, 2020 the EGFR p.L858R and p.V1010M were detected in a peripheral blood sample by 3D Medicines Inc. (Shanghai, China) using NGS and a digital droplet polymerase chain reaction (ddPCR) assay. Recombinant human endostatin (Endostar), pemetrexed, and nedaplatin were added because the efficacy was not significant. The lesion showed a tendency to progress even with afatinib replacement. Systemic bone imaging showed new lesions in the left side of the T11 vertebral, the left sacroiliac joint, and the right lower femur. Bilateral adrenal nodules $(25 \times 20 \mathrm{~mm}$ on the left, and $63 \times 37 \mathrm{~mm}$ on the right), and nodules in the upper inner quadrant of the left breast $(16 \times 11 \mathrm{~mm})$ were also found. Mesenchymal epithelial transition (MET) amplification and EGFR amplification were detected in a peripheral blood sample on February 19, 2020, and afatinib was discontinued after that. The patient died on June 20, 2020.

\section{Pedigree analysis}

\section{Proband}

The patient had no opportunity to undergo surgical treatment at the time of diagnosis. As the patient refused a needle biopsy, and there was insufficient tissue remaining from the sample obtained previously, peripheral blood was used for the ARMS assay (EGFR, ALK, and ROS1 gene hotspot detection), all of which were negative. Peripheral blood was then used for NGS detection on the 189-gene panel and ddPCR detection.

The EGFR p.L858R [mutant allele frequency (MAF): NGS, $0.07 \%$; ddPCR, $4.64 \%$ ] and the EGFR p.V1010M were detected by NGS (MAF: 49.59\%) and ddPCR (see Figure 2). Given the high MAF of V1010M, a germline mutation was considered, which the family analysis confirmed was a hybrid germline mutation.

\section{Family analysis}

Many family members suffer from lung cancer, and there is an obvious familial aggregation. We collected the blood samples of 9 relatives of the family and sent them to 3D Medicines Inc. for Sanger sequencing.

The verification site for the Sanger method was the EGFR p.V1010M. The chromosomal location was chr7: 55268962-55268962: G>A. The DNA range was chr7: 55268952-55268972, CATGGACGACG (>A) TGGTGGATGC. The PCR primers sequence was F: ACAGGCACCTGCTGGCAATA, and R: ATAGTGGACCTAAAAG

\section{GCTTACAATC.}

The sister (III1), daughter (IV4), and granddaughter (V1) of the proband (III3), and the daughter (IV1) and son (IV2) of the proband's sister were each heterozygous for the EGFR p. V1010M (see Figure 3).

\section{Protein structure and function prediction}

The effect of DNA level variation on an organism is ultimately manifested by a change in protein structure and function. The EGFR c.3028G>A caused the No. 1010 amino acids in the EGFR protein to change from valine $(\mathrm{V})$ to methionine (M). Using Schrodinger software to simulate the structure of EGFR protein, the results showed that the 1010th amino acid was not located in the kinase binding domain and near the $\mathrm{C}$ terminal (see Figure 4), and the variation to methionine had little effect on the structure of the EGFR dimer, which suggests that there might be other reasons for drug resistance (see Figure 5).

\section{Discussion}

With 1.38 million deaths every year, lung cancer is the most common cause of cancer deaths worldwide, and accounts for $18.2 \%$ of the total number of cancer deaths (7). With about 781,000 new cases and 626,000 deaths reported in 2014, in is also the cancer with the highest morbidity and mortality in China (8). Lung cancer has obvious familial aggregation, but it is not clear whether the same environment, similar habits, and inherent genetic factors all contribute to the familial aggregation of lung cancer.

A study involving 44,788 twins found that the heritability 

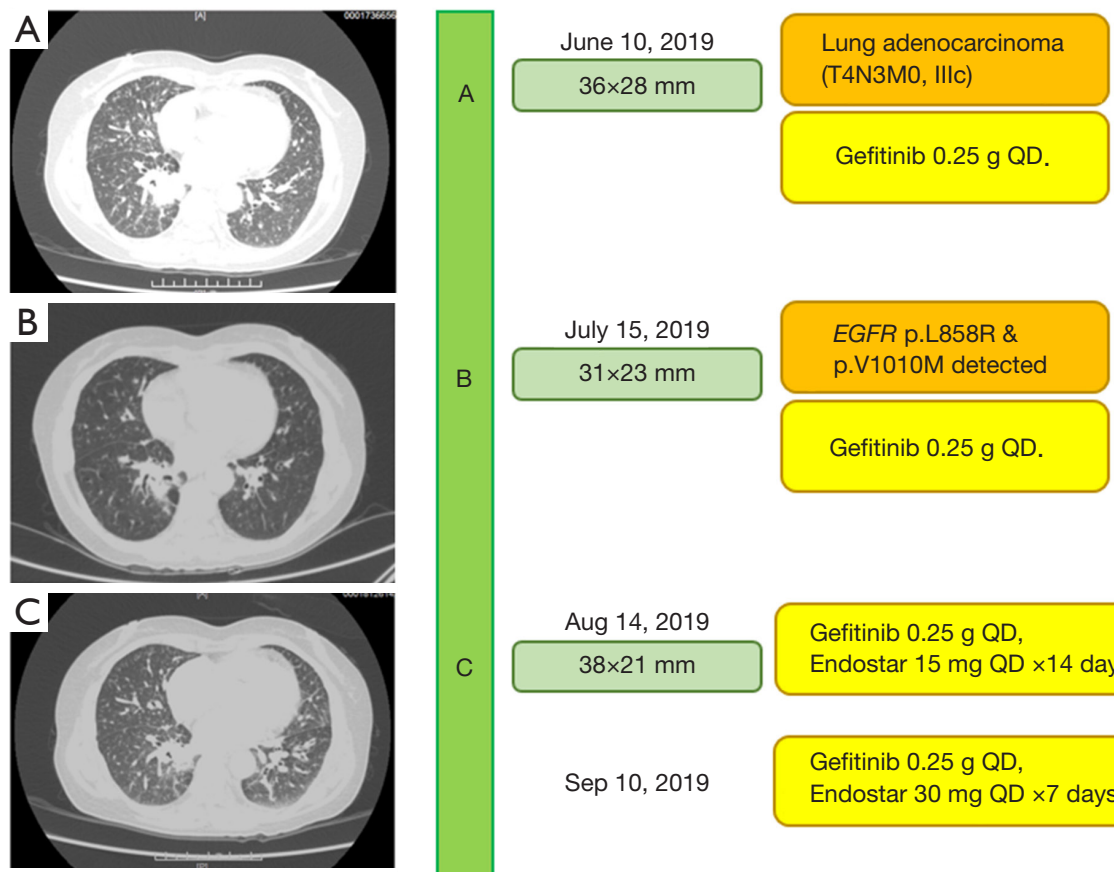

Gefitinib $0.25 \mathrm{~g}$ QD,

Endostar $15 \mathrm{mg}$ QD $\times 14$ days.

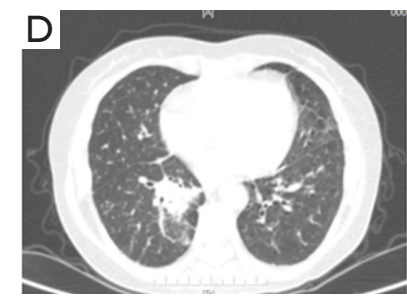

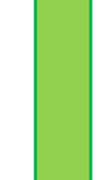

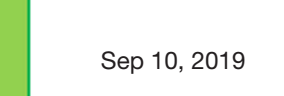

Gefitinib $0.25 \mathrm{~g} \mathrm{QD}$,

Endostar $30 \mathrm{mg}$ QD $\times 7$ days.
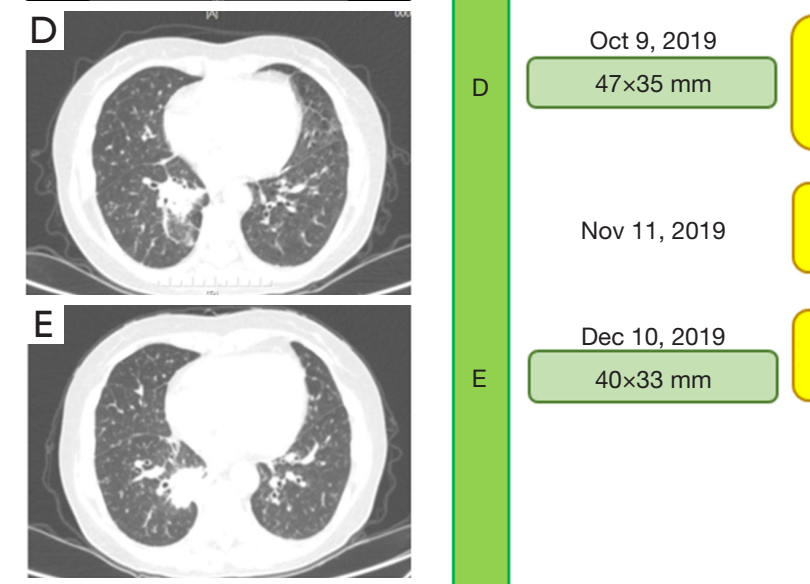

Gefitinib $0.25 \mathrm{~g}$ QD,

Endostar $30 \mathrm{mg} Q \mathrm{QD} \times 7$ days,

pemetrexed and nedaplatin.
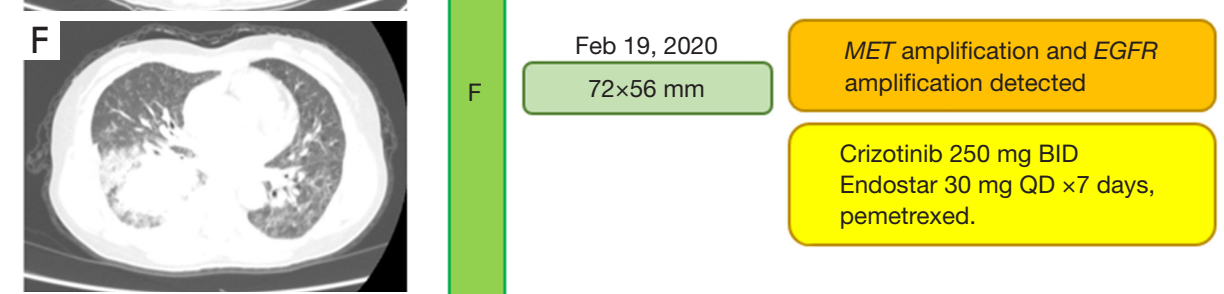

Afatinib $30 \mathrm{mg}$ QD,

Endostar $30 \mathrm{mg}$ QD $\times 7$ days.
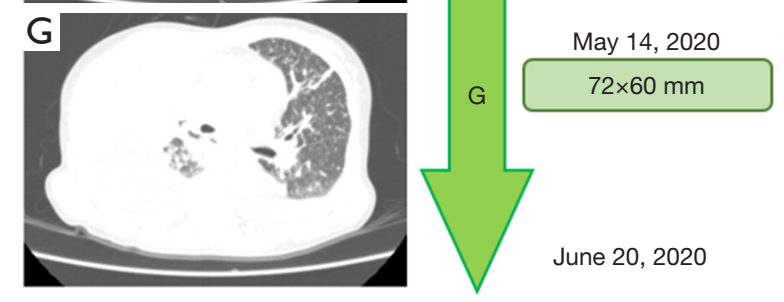

Crizotinib $250 \mathrm{mg}$ BID

Endostar 30 mg QD $\times 7$ days,

pemetrexed.

Afatinib $40 \mathrm{mg}$ QD,

Endostar $30 \mathrm{mg}$ QD $\times 7$ days.

June 20, 2020

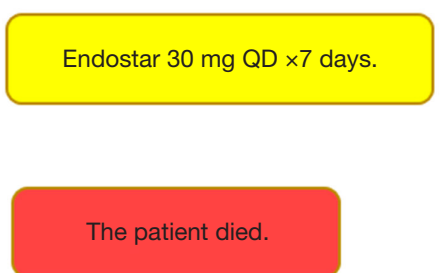

Figure 1 Chest computed tomography and treatment process of the proband (A-G). QD, every day; BID, twice a day; EGFR, epidermal growth factor receptor. 
A

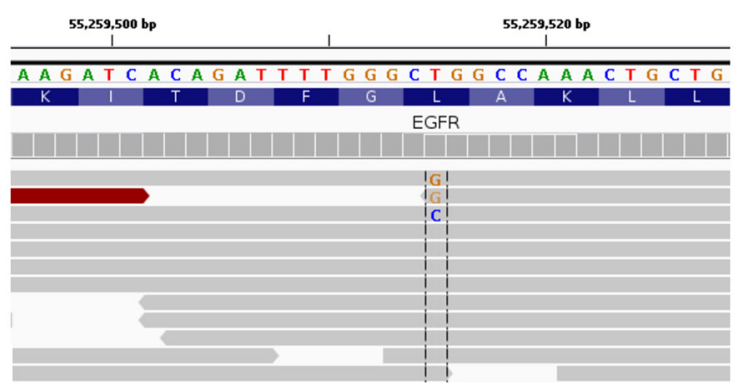

B

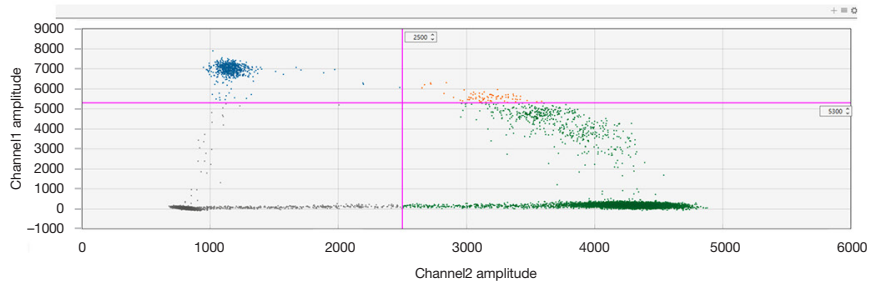

C

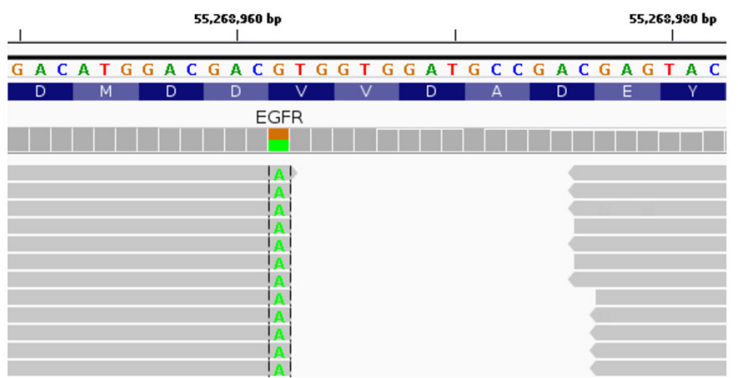

$\mathrm{D}$
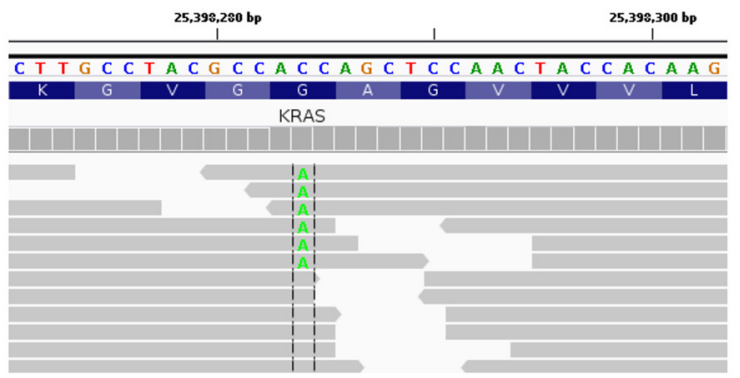

Figure 2 Detection results of the NGS and ddPCR. (A) Somatic mutation EGFR p.L858R of the proband by NGS. (B) Somatic mutation EGFR p.L858R of the proband by ddPCR. (C) Germline mutation EGFR p.V1010M of the proband by NGS; (D) Somatic mutation KRAS p.G12V of III4 by NGS. NGS, next-generation sequencing; ddPCR, digital droplet polymerase chain reaction; EGFR, epidermal growth factor receptor; KRAS, Kirsten rat sarcoma viral oncogene homologue.

of lung cancer was about $26 \%$ (2). This indicates that genetic factors only play a small part in the incidence of lung cancer, and it is mostly attributable to environmental factors. The results of a genome-wide association study (GWAS) also confirmed that the penetrance rate of gene variation was in direct proportion to the genetic risk of cancer: common single-nucleotide polymorphisms (SNPs) with low penetrance are associated with low genetic risk; infrequent moderate penetrance genetic variants are associated with moderate genetic risk, such as ataxia telangiectasia mutation $(A T M)$ and checkpoint kinase 2 (CHEK2); rare genetic variants with high penetrance are associated with high genetic risk [e.g., breast cancer $1 / 2(B R C A 1 / 2)$ are associated with hereditary breast and ovarian cancer], and mutL homolog 1 (MLH1), mutS homolog 2 (MSH2), and other mismatch repair (MMR) pathway gene variants are associated with Lynch syndrome (9). Notably, ATM, CHEK2, BRCA1/2, MLH1, and $M S H 2$ all belong to DNA damage repair (DDR) pathway genes. This type of genetic variation causes errors in the DNA replication process to go uncorrected, which causes more genes to mutate and tumors to eventually form. A GWAS showed that the genetic risk of most common cancers is polygenic (9); thus, DDR pathway gene germline mutations are the strongest genetic factors in tumor formation. However, as the incidence of DDR pathway variation in lung cancer is low, and there are only a few germline mutations, the role of genetic factors in the etiology of lung cancer has not yet been fully elucidated (10).

The EGFR is the most common driver gene of NSCLC, which suggests that the EGFR plays an important role in the occurrence of NSCLC. However, most of the pathogenic $E G F R$ mutations are somatic and are not passed on to the next generation. At present, very few EGFR germline mutations have been reported, but those that have been reported include G724S, K757R, V786M, T790M, L792F, R831H, V843I, L844V and D1014N (4,6,11). Among those, only T790M has been shown to have a significant family history, but the relationship between the T790M germline mutation and the occurrence of lung cancer has not been determined through family pedigrees (4). In a Japanese family, we have seen that the germline V843I mutation is a possible cause of lung cancer (6), but the same mutation in another Caucasian family has not been proven to be associated with lung cancer (11). Thus, the relationship between the V843I germline mutation and the occurrence of lung cancer has not yet been confirmed. Similar to this study, the V843I mutation was also resistant 
II3 EGFR wild type
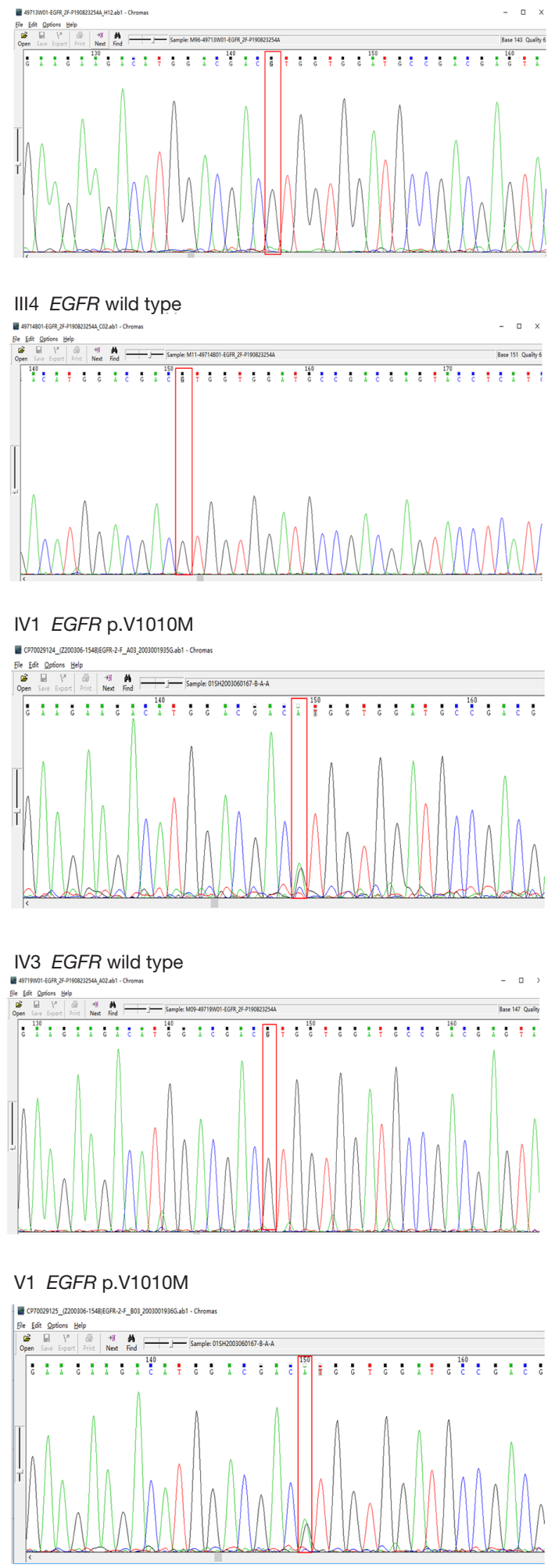

III1 EGFR p.V1010M
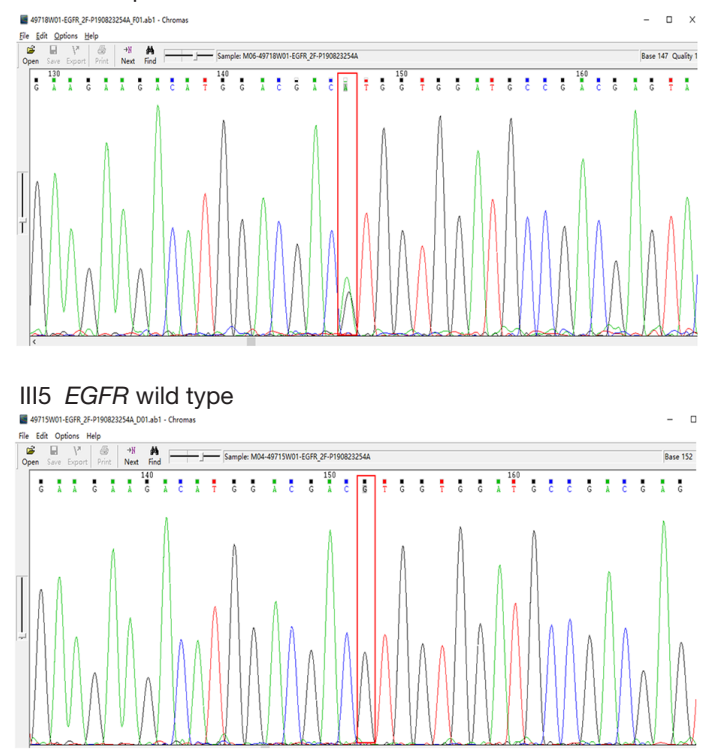

IV2 EGFR p.V1010M

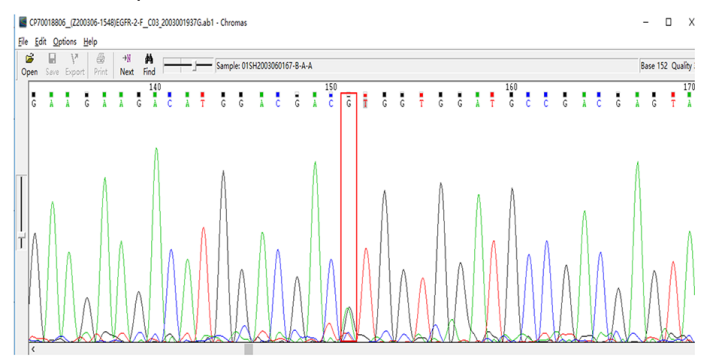

IV4 EGFR p.V1010M

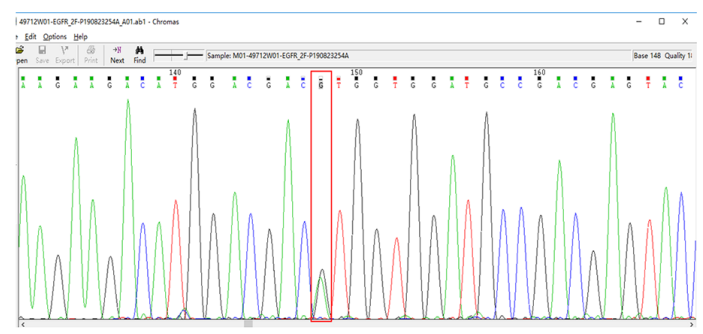

Figure 3 Detection results of familial samples by Sanger sequencing. EGFR, epidermal growth factor receptor. 


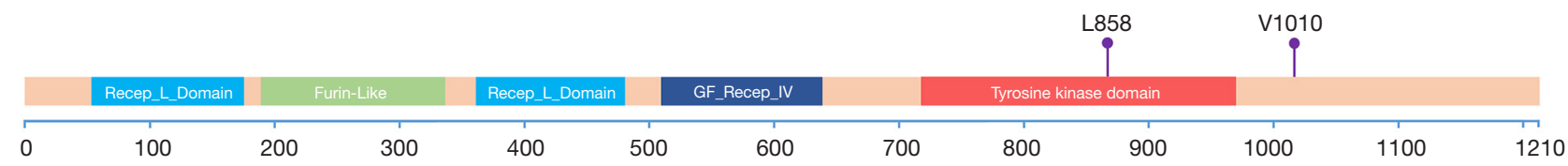

Figure 4 L858 and V1010 in relative positions of the EGFR protein. EGFR, epidermal growth factor receptor.
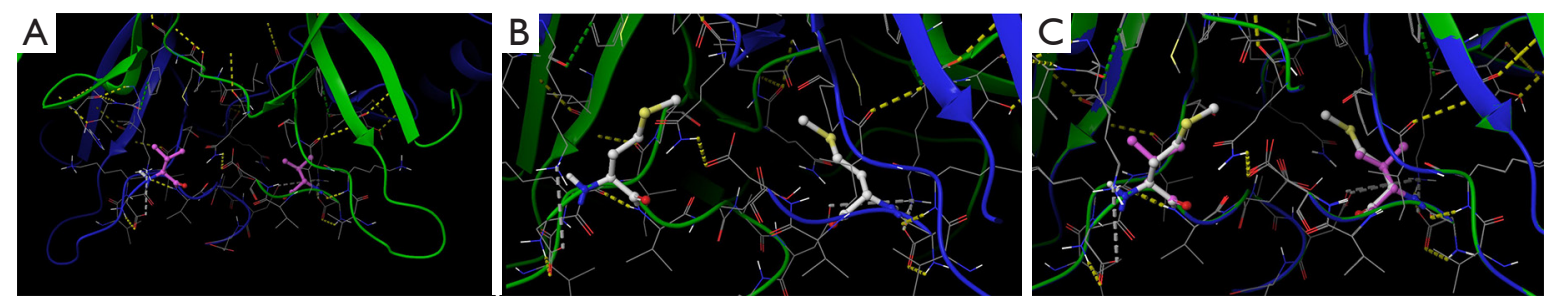

Figure 5 EGFR dimer structure simulated by Schrodinger software. (A) EGFR wild-type; valine in purple. (B) EGFR mutant; methionine in white. (C) Two structures compared in overlap. EGFR, epidermal growth factor receptor.

to erlotinib, and the protein structure analysis also revealed that the V843I mutation did not change the affinity of the ATP pocket region to EGFR-TKI; thus, the mechanism of resistance remains unknown (11).

The EGFR p.V1010M germline mutation was first identified in this family. This mutation had not been reported previously, and it is defined as a variant of unknown significance according to American College of Medical Genetics. On the Catalogue of Somatic Mutations in Cancer web site, the functional analysis through hidden Markov models predicted that the EGFR p.V1010M is pathogenic (score 0.72). In the pedigree (see Figure 6), it can be seen that among the 5 generations of the proband's maternal relatives, 7 are known to have developed into lung cancer with significant familial aggregation characteristics. Six of the 10 tested were found to be germline EGFR p.V1010M carriers. However, except for the proband (III3), no lung cancer was found in III1, IV1, IV2, IV4, and V1. Given that IV1, IV2, IV4, and V1 are all under the age of 40, long-term observation is required to determine whether they will develop lung cancer in the future. Whether the germline $E G F R$ mutation is related to the inheritance of lung cancer depends mainly on whether the germline mutation site is pathogenic. Thus, although we demonstrated that germline EGFR mutations could be passed on to the next generation, the current evidence does not confirm whether there is a causal relationship between the germline EGFR p.V1010M and the incidence of lung cancer.

Somatic mutations of the EGFR p.L858R and KRAS p.G12V (see Figure 2D) were found in the proband and II4, respectively. The EGFR p.L858R and KRAS p.G12V are obvious oncogenic driver gene variations. Since somatic variations are usually attributed to environmental influences in the later period, we have again confirmed that environmental factors are the main cause of lung cancer.

According to the different EGFR mutation sites, the efficacy of EGFR-TKIs also differs, and mutation sites can be divided into sensitive and resistance sites (1). In this case, the somatic mutation of EGFR p.L858R was simultaneously detected in addition to EGFR p.V1010M, which is a common sensitive mutation. However, after the use of gefitinib and afatinib, the optimal efficacy was SD as assessed by RECIST 1.1, and it showed a progression trend 4 months after the use of the drug, suggesting that EGFR p.V1010M may be the resistance site of EGFR-TKIs.

V1010 is located on exon 22 of the EGFR gene, near the kinase binding domain and the C-terminal. The threedimensional computer simulation of the structure of the mutant protein showed that the V1010M had little effect on the spatial structure of the EGFR dimer, and did not affect ATP pocket binding to the EGFR-TKI molecule. Thus, the biological mechanism of the EGFR-TKI resistance caused by the V1010M is still unknown, and further research needs to be conducted using animal models and cell lines.

Somatic variation caused by environmental factors is not usually thought to be directly passed on to the next generation. However, the same environment, similar habits, and even the same type of work in the same family can all cause the familial aggregation of cancer. Family history is still a risk factor for lung cancer. The risk of the disease may not be transmitted to offspring through DNA sequence changes of the germline, but the risk of the disease can 


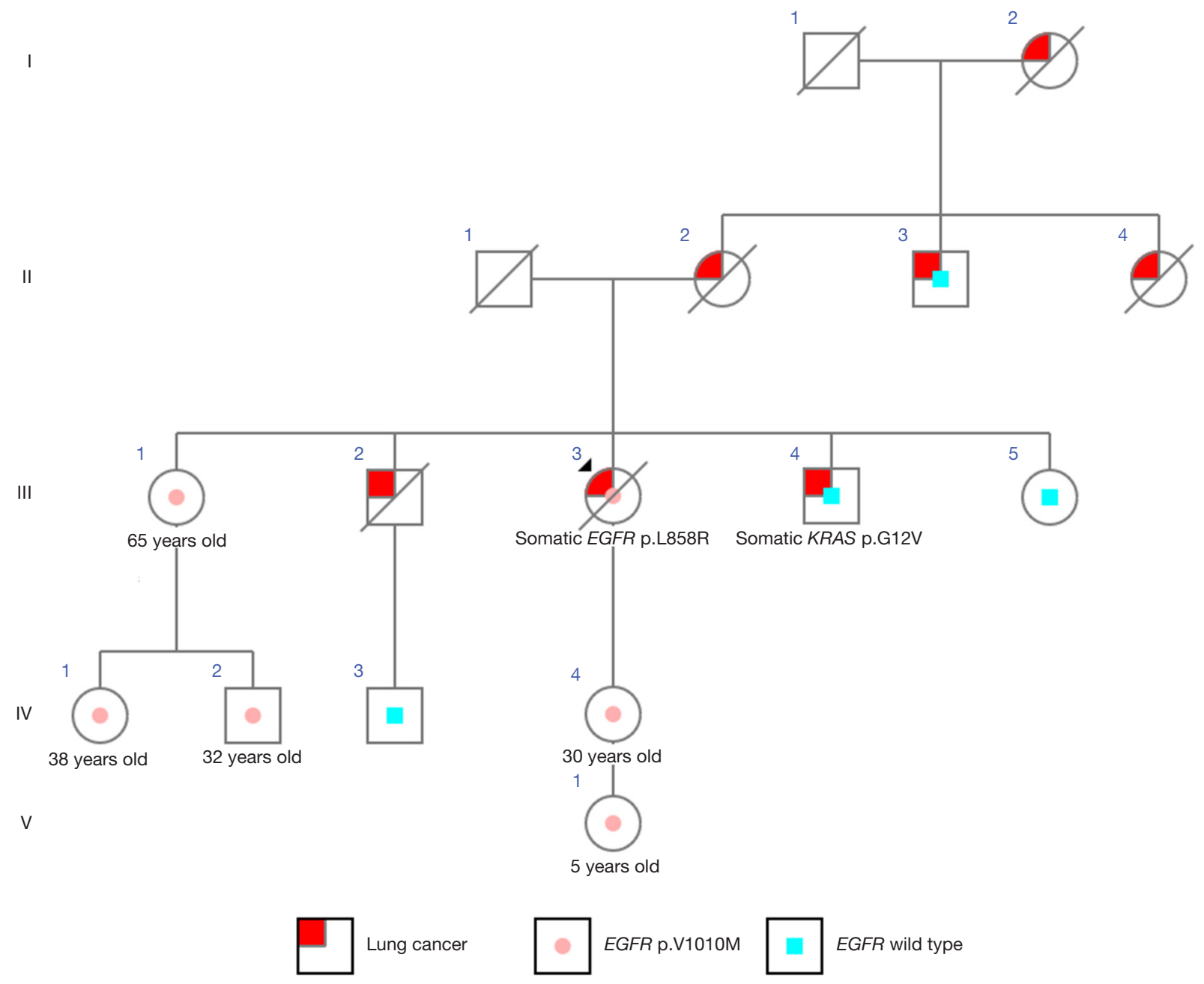

Figure 6 The pedigree analysis. EGFR, epidermal growth factor receptor.

also be increased through DNA methylation, histone modification, and ribonucleic acid regulation (10). Thus, the importance of family history assessments should not be underestimated. The family we reported on also confirm that people with a family history of lung cancer have a higher risk of developing lung cancer.

\section{Conclusions}

In summary, this family is the first family with the EGFR p.V1010M germline mutation to be analyzed by pedigree. This mutation site has not been proven to be a genetic factor for the occurrence of lung cancer, but it is likely a drugresistant mutation of EGFR-TKIs. Next, we will conduct cell-line and animal model studies to explore the biological mechanism of the EGFR p.V1010M in the development and treatment of disease resistance. We will also continue our long-term observation of this family to investigate the correlation between the EGFR p.V1010M and the development of lung cancer, which will also improve the awareness of cancer prevention and health care in this family, help with diagnosis, and enable timely interventions.

\section{Acknowledgments}

We thank Xudong Shen, Hushan Zhang, Xueke She and Dan Fu from 3D Medicines Inc. for their technical assistance in the operation of NGS detections and protein structure prediction.

Funding: This study was funded by the Senior Medical Talents Program of Chongqing for Young and Middle-Aged and Kuanren Talents Program of the Second Affiliated 
Hospital of Chongqing Medical University (grant No. KY2019Y003).

\section{Footnote}

Reporting Checklist: The authors have completed the MDAR reporting checklist. Available at https://atm.amegroups. com/article/view/10.21037/atm-21-6925/rc

Data Sharing Statement: Available at https://atm.amegroups. com/article/view/10.21037/atm-21-6925/dss

Conflicts of Interest: All authors have completed the ICMJE uniform disclosure form (available at https://atm.amegroups. com/article/view/10.21037/atm-21-6925/coif). DH is from the Medical Department, 3D Medicines Inc. All authors report that Xudong Shen, Hushan Zhang, Xueke She and Dan Fu from 3D Medicines Inc. offered their technical assistance in the operation of NGS detections and protein structure prediction. The authors have no other conflicts of interest to declare.

Ethical Statement: The authors are accountable for all aspects of the work in ensuring that questions related to the accuracy or integrity of any part of the work are appropriately investigated and resolved. All procedures performed in this study involving human participants were in accordance with the Declaration of Helsinki (as revised in 2013). The study was approved by the Ethics Committee of the Second Affiliated Hospital of Chongqing Medical University (No. 135, 2021) and informed consent was taken from all the participants.

Open Access Statement: This is an Open Access article distributed in accordance with the Creative Commons Attribution-NonCommercial-NoDerivs 4.0 International License (CC BY-NC-ND 4.0), which permits the noncommercial replication and distribution of the article with the strict proviso that no changes or edits are made and the original work is properly cited (including links to both the formal publication through the relevant DOI and the license). See: https://creativecommons.org/licenses/by-nc-nd/4.0/.

\section{References}

1. Dong J, Li B, Lin D, et al. Advances in Targeted Therapy and Immunotherapy for Non-small Cell Lung Cancer Based on Accurate Molecular Typing. Front Pharmacol 2019;10:230.

2. Lichtenstein P, Holm NV, Verkasalo PK, et al. Environmental and heritable factors in the causation of cancer--analyses of cohorts of twins from Sweden, Denmark, and Finland. N Engl J Med 2000;343:78-85.

3. Han B, Tjulandin S, Hagiwara K, et al. EGFR mutation prevalence in Asia-Pacific and Russian patients with advanced NSCLC of adenocarcinoma and nonadenocarcinoma histology: The IGNITE study. Lung Cancer 2017;113:37-44.

4. Lu S, Yu Y, Li Z, et al. EGFR and ERBB2 Germline Mutations in Chinese Lung Cancer Patients and Their Roles in Genetic Susceptibility to Cancer. J Thorac Oncol 2019;14:732-6.

5. Lemmon MA, Schlessinger J. Cell signaling by receptor tyrosine kinases. Cell 2010;141:1117-34.

6. Ohtsuka K, Ohnishi H, Kurai D, et al. Familial lung adenocarcinoma caused by the EGFR V843I germ-line mutation. J Clin Oncol 2011;29:e191-2.

7. Jemal A, Bray F, Center MM, et al. Global cancer statistics. CA Cancer J Clin 2011;61:69-90.

8. Chen WQ, Li H, Sun KX, et al. Report of Cancer Incidence and Mortality in China, 2014. Zhonghua Zhong Liu Za Zhi 2018;40:5-13.

9. Sud A, Kinnersley B, Houlston RS. Genome-wide association studies of cancer: current insights and future perspectives. Nat Rev Cancer 2017;17:692-704.

10. Coté ML, Liu M, Bonassi S, et al. Increased risk of lung cancer in individuals with a family history of the disease: a pooled analysis from the International Lung Cancer Consortium. Eur J Cancer 2012;48:1957-68.

11. Demierre N, Zoete V, Michielin O, et al. A dramatic lung cancer course in a patient with a rare EGFR germline mutation exon 21 V843I: Is EGFR TKI resistance predictable? Lung Cancer 2013;80:81-4.

(English Language Editor: L. Huleatt)

Cite this article as: Li N, Liu C, Xiong L, Huang D, Jiang Y. Pedigree analysis of the EGFR p.V1010M germline mutation in a family with a family history of non-small-cell lung cancer. Ann Transl Med 2022;10(3):154. doi: 10.21037/atm-21-6925 